\title{
Performances evaluation of four typical unmanned aerial vehicles used for pesticide application in China
}

\author{
Wang Shilin, Song Jianli, He Xiongkui*, Song Le, Wang Xiaonan, \\ Wang Changling, Wang Zhichong, Ling Yun
}

(College of science, China Agriculture University, Beijing 100193, China)

\begin{abstract}
In recent years, unmanned aerial vehicles (UAVs) for plant protection have achieved rapid development in China. In order to test and evaluate the performances of pesticides application and development status of UAVs in China, four typical UAV models were selected to test the spraying coverage, penetrability, droplets density and the work efficiency. The results showed that the deposition and spraying liquid coverage were inconsistent both in lateral and longitudinal direction. Under the condition of the similar amount of spray volume and operation parameters, the volume median diameter (VMD) of the droplet was negatively correlated with the coverage density. The failure of the UAVs for plant protection mainly took up on the blockage of nozzle, transfusion tube and the liquid pump. The failure rate of UAVs took up 3.73\%-4.36\% of the total working time. The operation of UAVs during ground service took up 50\% of the total working time, the preparation work took up $10 \%$, and the route planning took up around $10 \%$, while net operation time only took up around $30 \%$. On the whole, the high efficiency of UAV was not fully achieved; the daily operated area was not in a satisfactory level now. The spraying performances of UAVs still need further improvement.
\end{abstract}

Keywords: unmanned aerial vehicle, work efficiency, pesticide application, deposition, distribution

DOI: 10. 25165/j.ijabe.20171004.3219

Citation: Wang S L, Song J L, He X K, Song L, Wang X N, Wang C L, et al. Performances evaluation of four typical unmanned aerial vehicles used for pesticide application in China. Int J Agric \& Biol Eng, 2017; 10(4): 22-31.

\section{Introduction}

Plant protection is an important segment that

\section{Received date: 2017-01-18 Accepted date: 2017-06-12}

Biographies: Wang Shilin, PhD candidate, research interest: crop protection machinery and pesticide application, Email: shilinag@163.com; Song Jianli, PhD, Associate Professor, research interest: crop protection machinery and pesticide application, Email: songjianli170@163.com; Song Le, PhD candidate, research interest: pesticide residue analysis, Email: 435823956@qq.com; Wang Xiaonan, PhD candidate, research interest: pesticide application drift, Email: 404694775@qq.com; Wang Changling, $\mathrm{PhD}$ candidate, research interest: aerial pesticide application, Email: wcl1991@cau.edu.cn; Wang Zhichong, PhD candidate, research interest: crop protection machinery and pesticide application, Email: 549422839@qq.com; Ling Yun, PhD, Professor, research interest: synthesis of agrochemicals, Email: lyun@cau.edu.cn.

* Corresponding author: He Xiongkui, PhD, Professor, research interest: crop protection machinery and pesticide application. Centre for Chemicals Application Technology (CCAT), China Agricultural University, No. 2, Yuanmingyuan West Road, Beijing 100193, China. Email: xiongkui@cau.edu.cn. guarantees fertility and good harvest in agriculture. The equipment is an indispensable tool to realize the target ${ }^{[1]}$. At present, the plant protection machinery is primarily manual and half mechanized in China, which caused high labor intensity, low efficiency and high probability of poisoning incidents ${ }^{[2,3]}$. With the popularization of boom sprayer, the labor intensity has been reduced greatly and operating efficiency has been improved dramatically. In the mountainous areas, boom sprayers have encountered a lot of difficulties in field work. Agricultural aviation in these areas is thus dominated by small unmanned aerial vehicles (UAVs) ${ }^{[4]}$. Compared to conventional agricultural aircraft, UAVs do not require a special airport and can be operated by remote control ${ }^{[5]}$, which is very suitable for complex terrain that common ground machinery cannot enter. In addition, UAVs possess high work efficiency, and strong ability to deal with sudden disasters with low risk ${ }^{[6]}$. Also, UAVs can help effectively reduce the harm of pesticides to human 
and environmental pollution during the process of pesticides application $^{[7,8]}$. Therefore, researchers started to pay more attention to popularize UAVs in pesticide application in the past few years ${ }^{[9]}$.

In recent years, a lot of researches were conducted on UAVs, which mainly focused on the effect of operating parameters on the deposition of droplets and the biological efficiency, which provided a very useful foundation for agricultural aviation applications. Zhang et al. ${ }^{[10]}$ used the infrared thermal imager to study the distribution of spray droplets through temperature gradient of single rotor UAV, and the study indicated that the infrared thermal imaging technology can reflect the distribution regularities of droplets on rice accurately. $\mathrm{Ru}$ et al. ${ }^{[11]}$ studied on the aerial electrostatic spray and measured deposition with carbon paper and determined drift by eosin staining. It was found that electrostatic spray can improve the uniformity of droplets deposition, reduce pesticide drift, and increase the pesticide utilization and control efficiency. Qiu et al. ${ }^{[12]}$ studied the spraying performance of CD-10 UAV under the influence of flight height and velocity. A relevant model was established to clarify the relationship between deposition concentration, deposition uniformity, flight height and velocity. As a result, the study found that flight altitude, flight velocity and the interaction between two factors significantly affect the density of droplets and uniformity of droplet deposition. Gao et al. $^{[13]}$ studied the control efficiency of bifenthrin on wheat midge sprayed by single rotor electric UAV in low-altitude and it was proven that centrifugal nozzle is better than hydraulic nozzle. Qin et al. ${ }^{[14]}$ studied the influence of spraying parameters of N-3 UAV on droplets deposition of maize canopy and control effect of insecticides sprayed by UAV against plant hoppers, respectively. The results showed that both the insecticidal efficacy and the persistence period were greater than those achieved with a hand lance operated from a stretcher-mounted sprayer ${ }^{[4,14]}$. Moreover, there are also some studies related to atomization characteristics of UAV nozzles. Wen et al. ${ }^{[15]}$ studied the atomization characteristics of ultra-low-volume swirl nozzle for UAVs. $\mathrm{Ru}$ et al. ${ }^{[16]}$ analyzed droplet size distribution of aerial nozzle for plant protection in wind tunnel and flight conditions.

Despite these preceding studies, almost all of the researches focused on the effect of working parameters on droplet deposition and biological efficacy ${ }^{[17]}$. High efficiency, especially the strong ability to deal with sudden disasters including plant diseases and insect pests with low risk, is one of the most important reasons for the greatly developed UAVs. However, there is no report on the evaluation of the working efficiency of UAVs for plant protection as a very important evaluation index. As an emerging technology, there are still a series of practical issues for UAV spraying for pest protection ${ }^{[9]}$, such as uniformity of droplet distribution, droplet coverage ratio, penetrability of pesticide into the crop canopy, and working efficiency of UAV. In order to identify the pesticides application performances and development status of UAVs in China, four typical UAVs for plant protection, sold in domestic market and tested by the National Plant Protection Machinery Testing Center, were tested in this research.

The aims of this research were to explore the uniformity and coverage of droplet deposition in a multi-spraying swath; study the penetrability of pesticide into the canopy; evaluate the working efficiency of UAVs and to provide technical reference and guidance for a proper and safe aerial spraying in agricultural production of China.

\section{Materials and methods}

\subsection{Selection of four typical UAVs}

The four investigated UAVs included: a gas engine motive 3WQF120-12 single rotor UAV (Anyang Quanfeng Aviation Plant Protection Technology Co., Ltd.), a gas engine motive 3CD-15 single rotor UAV (Wuxi Hanhe Aviation Technology Co., Ltd.), a battery motive WSZ-0610 six rotors UAV (Shandong Wish Plant Protection Machinery Co., Ltd.), and a battery motive HY-B-15L single rotor UAV (Shenzhen high-tech new agricultural technology Co. Ltd.). The main technical parameters of UAVs are shown in Table 1. 
Table1 Primary technical parameters of selected UAVs

\begin{tabular}{|c|c|c|c|c|}
\hline Type & 3WQF120-12 & 3CD-15 & WSZ-0610 & HY-B-15L \\
\hline Cost per vehicle/Yuan RMB & 228000 & 250000 & 90000 & 188500 \\
\hline Rotor length/mm & 2410 & 2240 & 2220 & 2460 \\
\hline Tank capacity/L & 12 & 15 & 10 & 15 \\
\hline Number of nozzle & 2 & 4 & 2 & 5 \\
\hline Type of nozzle & LU120-02 & Flat-fan 01 & Centrifugal atomizer & Four flat-fan and one cone \\
\hline Type of pump & Diaphragm pump & Diaphragm pump & Gear pump and diaphragm pump & Diaphragm pump \\
\hline Full load flight time/min & 30 & 20 & 20 & 15 \\
\hline Unload flight time/min & 50 & 30 & 40 & 35 \\
\hline
\end{tabular}

\subsection{Experiment design}

The experiment was carried out in early May, 2016 in Anyang City, Henan Province (geographical longitude $114^{\circ} 35^{\prime}-114^{\circ} 59^{\prime} \mathrm{E}$, latitude $35^{\circ} 39^{\prime}-36^{\circ} 09^{\prime} \mathrm{N}$ ) with the meteorological conditions of field temperature $28.5^{\circ} \mathrm{C}$ $30.9^{\circ} \mathrm{C}$, wind speed $1.63-1.73 \mathrm{~m} / \mathrm{s}$ and relative humidity 41.4\%-54.7\%. Spraying uniformity, penetrability and work efficiency on wheat of these four typical UAVs were measured, respectively. The wheat was in grain-filling stage with a plant height of $60 \mathrm{~cm}$ and a row spacing of $20 \mathrm{~cm}$. The tests were divided into two parts. Firstly, the uniformity of deposition and coverage of droplets were determined in a specific field $(100 \mathrm{~m} \times$ $100 \mathrm{~m})$. Then, the tests of droplets penetrability in canopy and working efficiency were conducted under the large-scale pest prevention.

\subsubsection{Spray liquid and flight parameters}

During the whole period of tests, the flight parameters of the four UAVs were set up according to their daily actual filed practice, and the spray volume of each UAV was set as $12 \mathrm{~L} / \mathrm{hm}^{2}$. The dosage and pesticides used in the tests are set as follows: $60 \%$ imidacloprid SC $\left(90 \mathrm{~g} / \mathrm{hm}^{2}\right)$, $20 \%$ fenvalerate-malathion EC $\left(750 \mathrm{~g} / \mathrm{hm}^{2}\right), \quad 30 \%$ tebuconazole SC $\left(375 \mathrm{~g} / \mathrm{hm}^{2}\right)$ and $10 \%$ amino acids water soluble fertilizer $\left(375 \mathrm{~g} / \mathrm{hm}^{2}\right)$. In order to guarantee the comparability of spraying quantity of each model in the tests, the flow rate of nozzles were calibrated according to the flight parameters of the four models of UAVs. Specific flight parameters are shown in Table 2.

\subsubsection{Deposition and coverage of pesticide}

The spraying deposition, distribution and coverage of droplets were tested in a $100 \mathrm{~m} \times 100 \mathrm{~m}$ area. Filter paper and water sensitive paper (WSP) were used to analyze the deposition of liquid and the distribution of droplets in multi-spraying swath. Three lines of filter papers and WSPs were manipulated perpendicular to flight direction (lateral direction) in the center of testing region, and the length of each line was $15 \mathrm{~m}$. The interval of filter papers in line was $0.25 \mathrm{~m}$, and the interval of WSPs was $1.0 \mathrm{~m}$. The filter papers were placed horizontally, while the WPSs were placed in both horizontal and vertical direction. In order to test the effect of flight speed on the distribution uniformity, three longitudinal test zones were arranged along the route direction (longitudinal direction). Filter papers were placed horizontally in the distance of $1 \mathrm{~m}, 2 \mathrm{~m}, 5 \mathrm{~m}, 9 \mathrm{~m}$, $24 \mathrm{~m}, 34 \mathrm{~m}, 44 \mathrm{~m}$ and $50 \mathrm{~m}$ from the border. The height of filter papers and WSPs equals to wheat plants. The layout diagram of experimental field is shown in Figure 1, and the specific ways of filter papers and WSPs are shown in Figure 2.

\section{Table 2 Flight parameters of UAV in field test}

\begin{tabular}{lcccc}
\hline \multicolumn{1}{c}{ Type of UAV } & 3WQF120-12 & 3CD-15 & WSZ-0610 & HY-B-15L \\
\hline Flight velocity $/{\mathrm{m} \cdot \mathrm{s}^{-1}}^{\text {Flight height } / \mathrm{m}}$ & 5.0 & 6.0 & 4.0 & 4.5 \\
Swath/m & 2.0 & 2.0 & 2.0 & 1.5 \\
$\begin{array}{l}\text { Flow of single nozzle } \\
/ \mathrm{L} \cdot \mathrm{min}^{-1}\end{array}$ & 4.5 & 5.0 & 5.0 & 6.0 \\
\hline
\end{tabular}

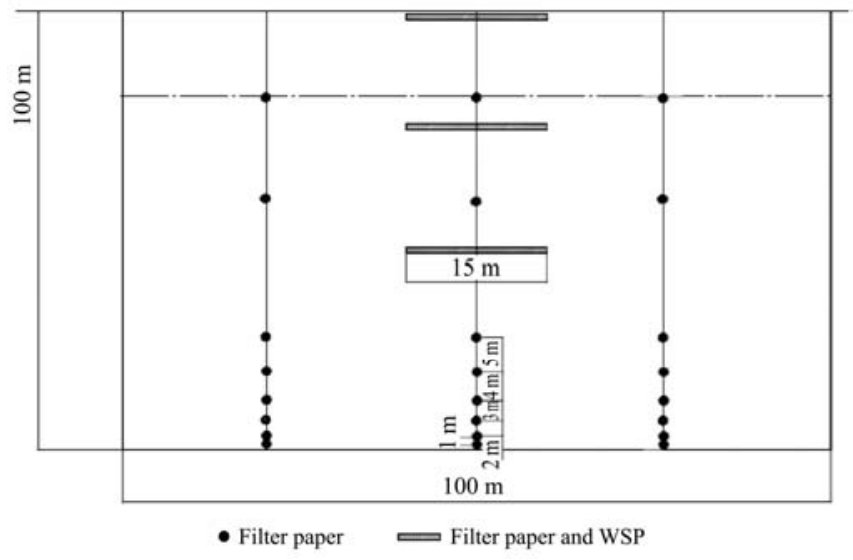

Figure 1 Layout of experimental field 


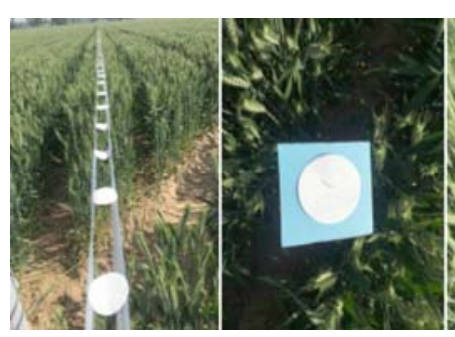

a. Crosswise filters b. Longitudinal filter

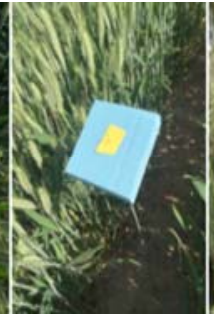

c. Horizontal WSP

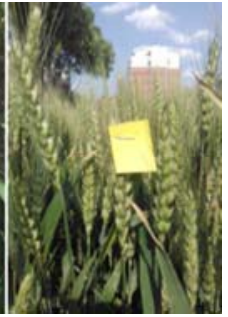

d. Vertical WSP
Figure 2 Diagram of filter papers and WSPs

\subsubsection{Penetrability of spraying liquids}

Testing plot was set in the real pest and disease control area of wheat field. Five sampling points were diagonally arranged in the control area of the four UAVs. Two hours after pesticide application, no less than $1 \mathrm{~kg}$ wheat plants of over ground part were collected in each sampling point, and equally divided into upper, middle and lower parts, then stored at $-20^{\circ} \mathrm{C}$ until analysis (storage time was not more than one week before analysis).

\subsubsection{Working efficiency}

Each operation team consists of two UAVs and three labors. One of the UAVs was used for pesticide application, and the other was a standby. Two labors controlled the UAVs in turns, and the other one was in charge of ground service. The working time of each operation team was from $6: 00$ to $10: 00$ and $15: 00$ to 19:00 every day. The experiment was carried out in the form of large-scale pest prevention. The farmland was assigned randomly to each operation team participated in this test. The following operation items of UAVs for each team were monitored three days in details. The operation items included the time of preparation, route planning, failure maintenance, ground service, net operation and the area operated.

\subsection{Sample collection and data processing}

\subsubsection{Filter paper sampling}

The fancy red with mass fraction of $1.5 \%$ was used as a tracer added into spraying liquid to quantify the amount of spray liquids deposited on the filter papers. After pesticide application, each filter paper was removed and then placed in separate sealed bags, and then stored in a cool and dark place before analysis. The collected filter papers were washed by $10 \mathrm{~mL}$ deionized water in the laboratory. After vibration and elution, $200 \mu \mathrm{L}$ of eluent was transferred into the ELISA plate, and scanned at
$492 \mathrm{~nm}$ by Multiskan MK3 enzyme micro-plate reader (Thermo scientific, USA) to estimate the concentration of fancy red and figure out the deposition of spraying liquids on unit area.

\subsubsection{WSP sampling}

After pesticide application, the WSPs were allowed to dry and placed into the labeled envelopes, then sealed in dry place. Rubber gloves are indispensable during the process of collecting WSPs. A 600 dpi digital image of each WPS was acquired with a handheld scanner in the lab. After that, an imagery software DepositScan ${ }^{[18]}$ (USDA, USA) was utilized to extract droplet deposits in the digital image and analyze the coverage, density, size of droplets on WSPs.

\subsubsection{Wheat sampling}

In order to analyze wheat samples, three layers of wheat plant were grinded into small pieces or powder by a vegetation disintegrator, respectively. Five grams of the sample was weighted into a $50 \mathrm{~mL}$ Teflon tube, while $5 \mathrm{~mL}$ of deionized water and $10 \mathrm{~mL}$ of acetonitrile were added as well. Five minutes later, samples were shaken in a reciprocating shaker for $10 \mathrm{~min}$. Then $4 \mathrm{~g}$ of $\mathrm{MgSO}_{4}$ and $1 \mathrm{~g}$ of $\mathrm{NaCl}$ were added with quick shake and swirled for $1 \mathrm{~min}$. Samples were centrifuged at 4000 $\mathrm{r} / \mathrm{min}$ for $5 \mathrm{~min}$, and the supernatant was transferred into a $1.5 \mathrm{~mL}$ centrifuge tube with $30 \mathrm{mg}$ GCB and $50 \mathrm{mg}$ PSA. The tube was swirled for $1 \mathrm{~min}$, then centrifuged at $20000 \mathrm{r} / \mathrm{min}$ for $1 \mathrm{~min}$. Transfer the supernatant solution into a sample vial for the LC-MS/MS analysis after filtration through a $0.22 \mu \mathrm{m}$ nylon filter. Tebuconazole was applied as the target pesticide to analyze the penetrability of spraying liquids by UAVs.

The LC-MS/MS analysis was carried out by using the Thermo TSQ Quantum triple-quadrupole mass spectrometer (Thermo Fisher, USA), which was equipped with an electro spray ionization (ESI) source and Surveyor Liquid Chromatography System. The separations were performed by using a $100 \times 2.1 \mathrm{~mm} \times$ $1.7 \mu \mathrm{m}$ Hypersil GOLD C18 analytical column from Thermo (USA). Elution was performed with 70\% acetonitrile as mobile phase A and $30 \%$ ultrapure water contained $0.1 \%$ formic acid as mobile phase $\mathrm{B}$. Separation of the analyzed from the C18 column was 
performed at a flow rate of $0.3 \mathrm{~mL} / \mathrm{min}$. The column was kept at $30^{\circ} \mathrm{C}$. The injected sample volume was $5 \mu \mathrm{L}$. Analysis of the compound was carried out by using the multiple reaction monitoring (MRM) mode and positive ESI mode. The conditions for MS detection as follows: electrospray voltage was $3500 \mathrm{~V}$; ionization and capillary temperature was $350^{\circ} \mathrm{C}$; atomization gas and curtain gas for high-purity nitrogen; the collision gas was argon with the pressure of $0.2 \mathrm{~Pa}$; sheath gas flow rate was $6.5 \mathrm{~L} / \mathrm{min}$; and auxiliary gas flow rate was $5.0 \mathrm{~L} / \mathrm{min}$. The other MRM conditions are shown in Table 3.

Table 3 MRM parameters of tebuconazole

\begin{tabular}{cccccc}
\hline Compound & Formula & $\begin{array}{c}\text { MRM } \\
\text { transitions } \\
/(\mathrm{m} / \mathrm{z})\end{array}$ & $\begin{array}{c}\text { Collision } \\
\text { energy } \\
\mathrm{E} / \mathrm{eV}\end{array}$ & $\begin{array}{c}\text { Tube } \\
\text { lens } \\
\mathrm{U} / \mathrm{V}\end{array}$ & $\begin{array}{c}\text { Retention } \\
\text { time } t \\
/ \mathrm{min}\end{array}$ \\
\hline \multirow{2}{*}{ Tebuconazole $\mathrm{C}_{16} \mathrm{H}_{22} \mathrm{ClN}_{3} \mathrm{O}$} & $\begin{array}{c}307.9 / 70.1, \\
307.9 / 151.0\end{array}$ & $18 / 25$ & $129 / 129$ & 1.86
\end{tabular}

\section{Results and discussion}

\subsection{Deposition and coverage of pesticide}

In order to calculate the spraying deposition, a standard curve was built by measuring the absorbance of a series of fancy red solutions of known concentrations. The mass fractions of standard solutions used in this test as follows: $0,5.0 \mathrm{mg} / \mathrm{kg}, 10.0 \mathrm{mg} / \mathrm{kg}, 15.0 \mathrm{mg} / \mathrm{kg}$, $20.0 \mathrm{mg} / \mathrm{kg}, 25.0 \mathrm{mg} / \mathrm{kg}, 50.0 \mathrm{mg} / \mathrm{kg}$ and $100.0 \mathrm{mg} / \mathrm{kg}$. The deposition of spraying liquids was quantified using the following linear Equations: $Y=0.0253 x+0.0317$ $\left(R^{2}=0.9982\right)$. In this Equation, $Y$ represents the absorbance obtained from ELISA plate; $x$ represents the concentration $(\mathrm{mg} / \mathrm{kg})$ of standard solutions. The absorbance of filter paper eluate was taken into the linear regression Equation to determine the content of fancy red in that eluate, and then the deposition of spraying liquids on unit area can be calculated by the fancy red concentration.

The deposition results in lateral direction of each UAV are shown in Figure 3. In the length of multi-spraying swath $(15 \mathrm{~m})$, there were obviously inconsistent amount of deposition among the four UAVs in different locations, and the maximum amount of deposition was several times of minimum on each UAV. Uniformity of pesticide distribution is an important factor in evaluating the effect from agricultural airplane ${ }^{[19]}$. In this case, the coefficient of variation $(\mathrm{CV})$ is used to evaluate the uniformity of spraying liquid deposition. The smaller the $\mathrm{CV}$ is, the better the uniformity of the spray deposition is ${ }^{[20]}$. The CVs of the deposition in lateral direction of the four UAVs respectively were $65.45 \%, 62.58 \%, 70.81 \%$ and $43.04 \%$, which were much greater than the CV (20\%) of boom sprayer specified in the Chinese National Standard ${ }^{[21]}$. The result obtained in this study was consistent with the conclusion of Qin et al. $^{[4]}$ in paddy and Zhang et al. ${ }^{[22]}$ in citrus, which indicated that there was a great uneven pesticide deposition sprayed by UAVs in China. Uneven spraying liquid distribution was determined by the operating parameters of UAVs and properties of spraying liquids. The spraying height of small UAVs can influence the distribution of droplets ${ }^{[22]}$. The flight height of four UAVs ranged from $1.5 \mathrm{~m}$ to $2.0 \mathrm{~m}$, which was much higher than that of ground plant protection machinery. Hence, the settling time and distance of droplets increased. Bird et al. ${ }^{[23]}$ indicated that the droplet size significantly affects the performance of aerial applications. The droplet size of four UAVs was smaller than ground pesticide application, which increased the specific surface area of spraying liquid and led to a more susceptible liquid to meteorological conditions. Therefore, drift and evaporation of droplets were further intensified in the process of settlement. In addition, the airflow created by UAVs can also affect the distribution uniformity of liquids in the process of droplets settlement.

The deposition results in longitudinal direction of four UAVs are shown in Figure 4. As can be seen from the figure, the deposition at the boundary of operation area was significantly higher than that of the central area. The acceleration and deceleration of the four UAVs near the boundary had a great influence on the uniformity of spraying liquids in longitudinal direction. The length of regulating velocity is about $10 \mathrm{~m}$. When the distance to boundary was greater than $10 \mathrm{~m}$, the amount of deposition is relative uniform in three longitudinal test lines due to the constant-speed movement of the UAVs. The pesticide application of UAVs is mainly in a low 
volume (LV) and ultra-low volume (ULV) spraying, thus the concentration of pesticide is particularly high. The

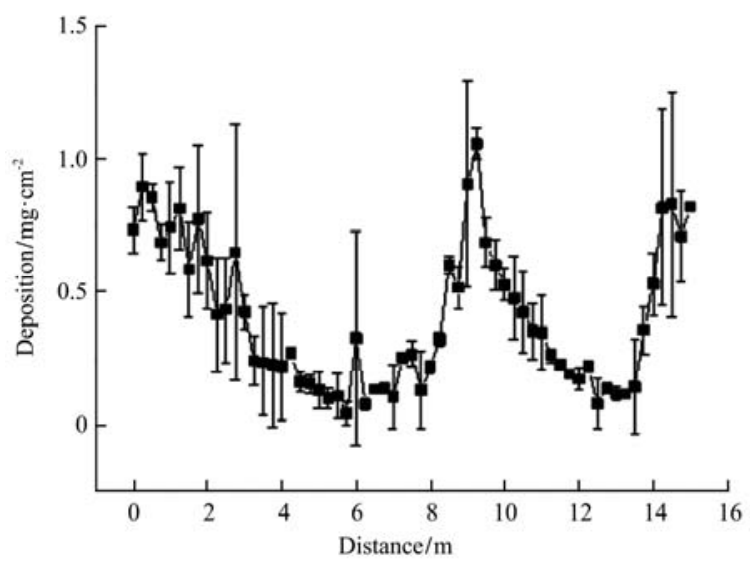

a. 3WQF120-12

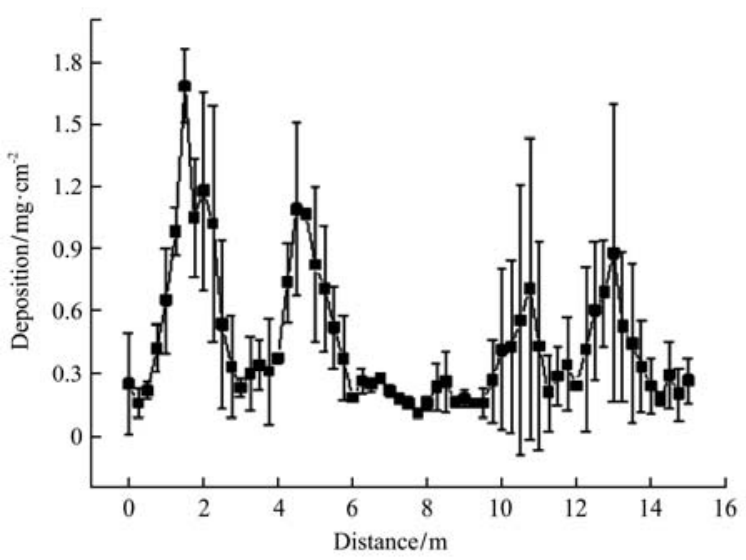

c. WSZ-0610 excess deposition of spraying liquids near the boundary is likely to cause crop injury or excessive pesticide residues.

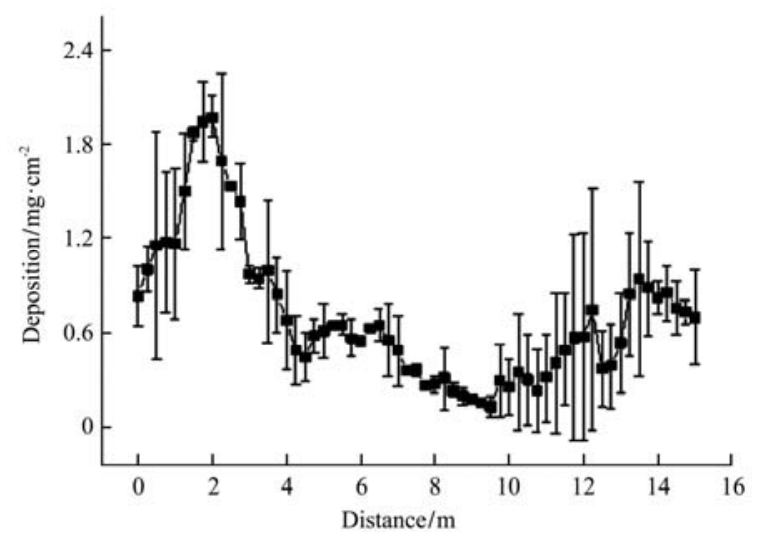

b. 3CD-15

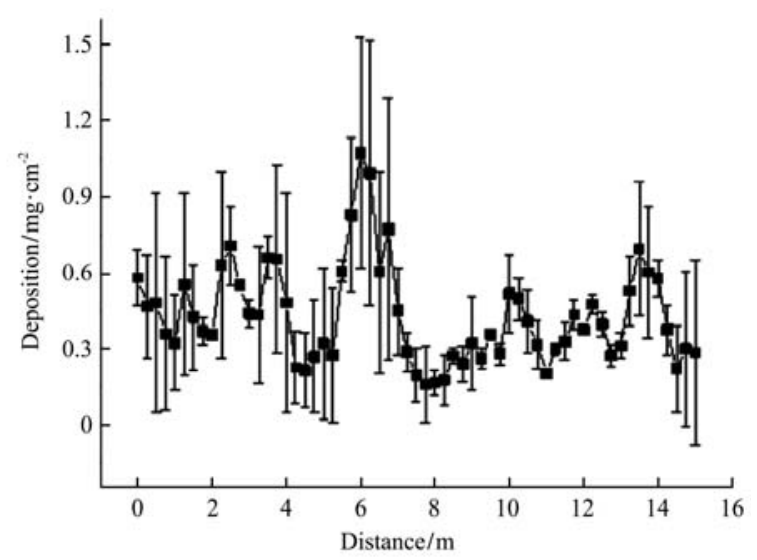

d. HY-B-15L

Figure 3 Deposit characters in crosswise of four UAVs
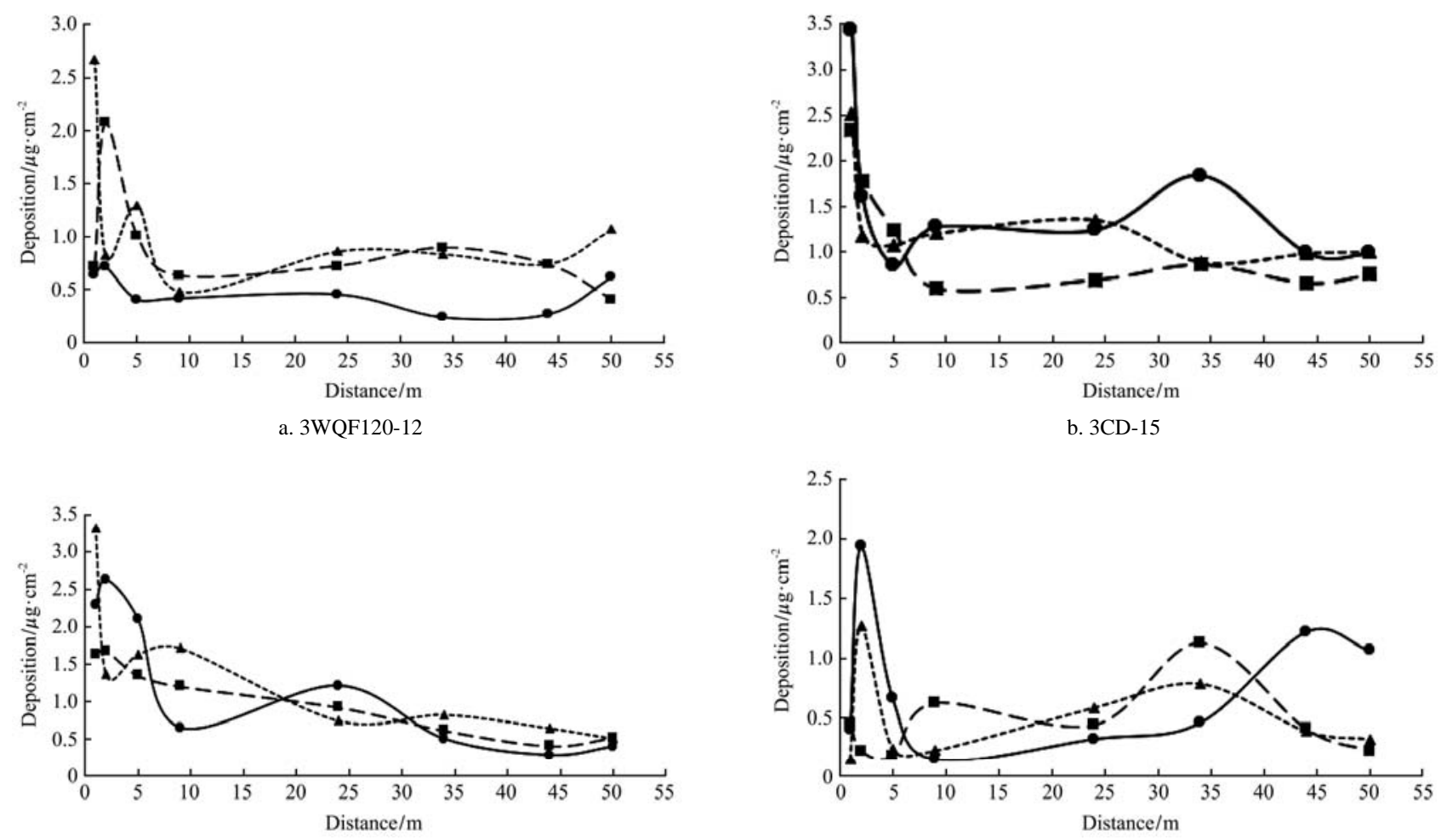

c. WSZ-0610

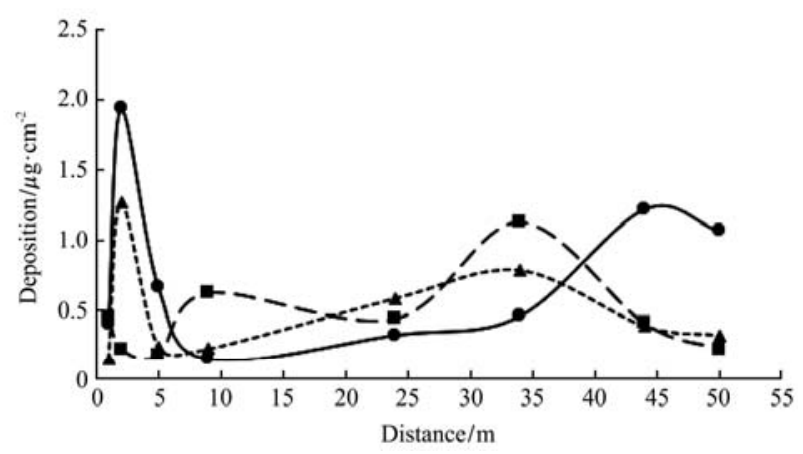

d. HY-B-15L

Figure 4 Deposit characters in longitudinal of four UAVs 
The comprehensive effects of flight parameters, properties of spraying liquids and meteorological conditions resulted in the un-uniformity of deposition applied by UAVs both in lateral and longitudinal direction. Thus, the uniformity of deposition can be enhanced by improving flight parameters, optimizing flight stability and operability. Moreover, changing the properties of spraying liquids, such as developing specialized formulations of aerial pesticide application or adding anti-drift and anti-evaporation adjuvants into the spraying liquids, can also help ${ }^{[24]}$.

The DepositScan software was used to measure the droplet coverage, droplet density, the variation between droplet size and droplet density on WSPs, and the results are shown in Table 4. Though both droplets density and coverage on vertical WSPs were lower than those on horizontal direction, there still existed certain amount of droplets. A downwards flow was produced when the rotors of UAVs were rotating, which helped the droplets to deposit on vertical targets through dispersing the canopy. The droplets density of WSZ-0610 on horizontal and vertical WSPs ranged at $8.2-127.2 \mathrm{~g} / \mathrm{cm}^{2}$ and $3.9-109.5 \mathrm{~g} / \mathrm{cm}^{2}$, respectively. The droplets' VMD of WSZ-0610 on the WSPs was $128 \mu \mathrm{m}$, which was the smallest because of a centrifugal nozzle. However, other droplets' VMDs from UAVs with hydraulic nozzles were much larger than those produced by centrifugal nozzle. The sizes of droplets were decided by nozzles of UAVs. With the same spray volume and similar operating parameters, the size of droplets and droplet density showed a negative correlation, while there was no such correlation between the percentage of coverage and droplet size. Although WSZ-0610 had the highest density of droplets on WSPs, its CV of deposition on crosswise reached $70.81 \%$, which was the highest of all. Smaller droplets were impressionable to the meteorological conditions ${ }^{[25,26]}$, and the air flow produced by six rotors of WSZ-0610 was different from that produced by single rotor UAVs. All these effects are possible to lead to the largest CV. However, further research for the specific reasons of imparity is still needed.

Table 4 Sizes of droplets, density and coverage on WSPs

\begin{tabular}{|c|c|c|c|c|c|c|c|}
\hline \multirow{2}{*}{ Type of UAVs } & \multicolumn{2}{|c|}{ Horizontal } & \multicolumn{2}{|c|}{ Vertical } & \multicolumn{3}{|c|}{ Sizes of droplets/ $\mu \mathrm{m}$} \\
\hline & Density/g $\cdot \mathrm{cm}^{-2}$ & Coverage/\% & Density $/ \mathrm{g} \cdot \mathrm{cm}^{-2}$ & Coverage/\% & DV(10) & DV(50) & DV(90) \\
\hline 3WQF120-12 & $6.9-68.1$ & $0.48-2.21$ & $1.9-29.0$ & $0.13-1.64$ & 143 & 253 & 356 \\
\hline 3CD-15 & $6.6-26.9$ & $0.43-2.62$ & $2.4-16.7$ & $0.09-1.28$ & 157 & 314 & 543 \\
\hline WSZ-0610 & $8.2-127.2$ & $0.18-1.85$ & 3.9-109.5 & $0.12-1.08$ & 77 & 128 & 169 \\
\hline HY-B-15L & $14.7-38.5$ & $0.78-2.24$ & $2.9-21.6$ & $0.16-1.16$ & 133 & 264 & 340 \\
\hline
\end{tabular}

\subsection{Penetrability of spraying liquids}

In the process of determining the penetrability of tebuconazole, the external standard method was used for quantitative analysis. The test was conducted by fortifying a certain amount of standard solutions of tebuconazole into the blank substrate. Seven concentrations of standard solutions were set for the test, and they are $0,5.0 \mu \mathrm{g} / \mathrm{kg}, 10.0 \mu \mathrm{g} / \mathrm{kg}, 50.0 \mu \mathrm{g} / \mathrm{kg}$, $100.0 \mu \mathrm{g} / \mathrm{kg}, 500.0 \mu \mathrm{g} / \mathrm{kg}$ and $1000 \mu \mathrm{g} / \mathrm{kg}$. A linear regression equation was obtained through the determination of the base objects. Amount of tebuconazole in each layer was quantified by using the following linear equation: $Y=2.0048 x-1.334$ with $R^{2}=$ 0.9998. In this equation, $Y$ represents the response peak area obtained from analytical instrument, and $x$ represents the amount $(\mu \mathrm{g} / \mathrm{kg})$ of tebuconazole. The recoveries of added tebuconazole ranged from $86.67 \%$ to $88.73 \%$ with acceptable relative standard deviations (RSDs) of $0.90 \%$.

The amount of tebuconazole on upper layer of each UVA was defined as $100 \%$, while the quantity of tebuconazole on middle and lower was expressed in the percentages of upper layer. The results of penetrability of four UAVs are shown in Figure 5. WSZ-0610 and HY-B-15L had better penetrability. The depositions of tebuconazole sprayed by HY-B-15L on middle and lower were $46.8 \%$ and $16.4 \%$ of the upper layer, and the deposition of WSZ-0610 was $52.1 \%$ and $12.0 \%$, respectively. The depositions of tebuconazole sprayed by 3CD-15 on middle and lower were $39.0 \%$ and $10.4 \%$ of the upper layer. In the same way, the results of 
3WQF120-12 were $24.1 \%$ and 5.2\%, respectively. Leaf area index (LAI), structure of canopy and natural wind exerted a tremendous influence on pesticide deposition and distribution in crop canopy ${ }^{[22,27,28]}$. However, when the three factors are parallel, the impact of flight parameters on penetrability will become more pronounced $^{[4]}$. Table 2 shows the flight parameters of UVAs, the speeds of 3WQF120-12 and 3CD-15 were $5 \mathrm{~m} / \mathrm{s}$ and $6 \mathrm{~m} / \mathrm{s}$, respectively, which were faster than the speeds of WSZ-0610 and HY-B-15L. The airflow on wheat canopy created by UAVs will be changed by the flight speed. The flight height of HY-B-15L was $1.5 \mathrm{~m}$, which was lower than the other three, and led a larger airflow on wheat canopy. Small droplets are more sensitive to meteorological conditions ${ }^{[29]}$ so that they are more vulnerable to winds. As can be seen in Table 4, the VMD of WSZ-0610 was the smallest for $128 \mu \mathrm{m}$, which was more susceptible to the transfer of downward air stream. Also, the airflow of six rotors UAV WSZ-0610 was different from that of single rotor UAVs. Therefore, the penetrability of WSZ-0610 and HY-B-15L were better than 3WQF120-12 and 3CD-15.

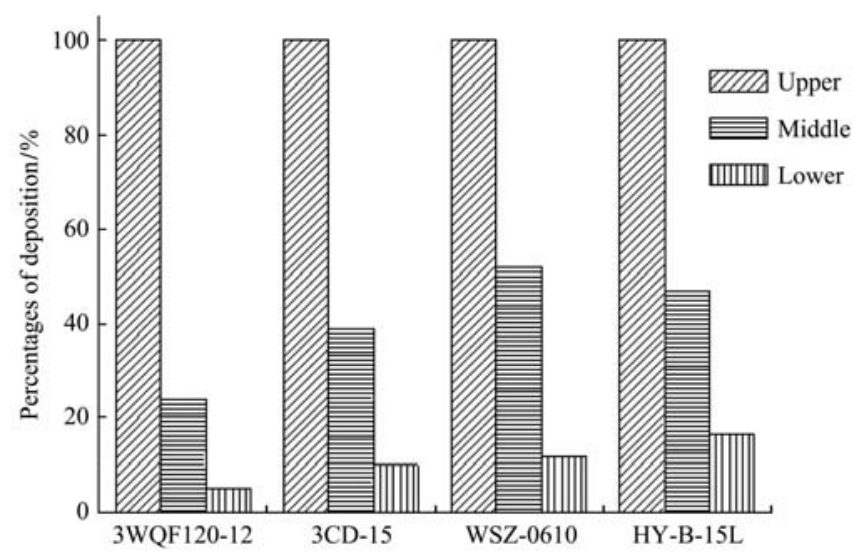

Figure 5 Deposition of four UAVs in different layers

\subsection{Working efficiency of UAV}

As a high efficiency plant protection machinery, the statistics of working efficiency and the time scale of every operation item make a great deal of sense. However, working efficiency of UAV was simply evaluated by area completed per sortie in previous. The impact of failure maintenance, preparation and ground service were neglected. In this study, preparation included the assemble of UAVs and preparation of spraying liquid, and ground service included the replacement of spraying liquid and fuel oil or battery. Failure rate is the ratio that the time of the failure took up during the whole working process. The failure included not only the damage and replacement of parts, but also the stability of control system, the blocking of pump, tube, nozzle, and all other factors which will influence the normal work stability. Net operation is the process of UAVs spraying in wheat field.

The percentages of operation items and daily operated area of UAVs are shown in Table 5. The proportion of preparation time was relatively balanced, ranging from $7.60 \%$ (3CD-15) to $9.56 \%$ (3WQF120-12), and is mainly at the beginning of the work. Since there was no crash or other major failures occurred in this test, the failures of the UAVs mainly occurred in the blocking of nozzles, transfusion tube and pump, and it took up 3.73\%-4.36\% of the total time during the process of crop protection. Those failures can be avoided via changing components and washing them with clean water. On the other hand, it indicated that the formulations of pesticide used were not suitable for the spray system of UAVs. The stability of the spray system can be improved by reducing the viscosity of the spraying liquids and increasing the fluidity or developing specialized formulations of aerial pesticide application. The observation of terrain (route planning) was around 10\%, which can be shortened through autonomous route planning and sortie. For example, a route planning algorithm with the minimum return number was proposed by $\mathrm{Xu}$ et al. ${ }^{[30]}$, which can reduce the ineffective energy consumption in non-operate situation and improve operational efficiency in the same time.

The percentages of ground service, net operation and daily operated area of HY-B-15L were not given because the motor of HY-B-15L generated a lot of heat during working. So HY-B-15L took a mode of two UAVs spraying alternately. When an UAV completed a sortie, it should be cooled for a while, and the ground staff can add pesticide in and change the batteries at the same time. Meanwhile, the other operator can control the other UAV to spray as well. Also, the takeoff and landing time of UAVs was saved and the time for ground staff to leave the operating area can be saved as well. However, this 
spraying mode greatly increased operating cost.

On the whole, the proportion of ground time accounted $50 \%$ of the whole process, while the net operation was only about 30\%. The ground service of 3CD-15 was the highest, reaching $52.14 \%$. This was because the power system and spray system of 3CD-15 was independent, so that the spraying system was supplied with additional power from the battery. Hence, batteries should be changed besides adding pesticide and fuel in ground service for 3CD-15. In this test, the daily working area ranged from $13.4 \mathrm{hm}^{2}$ (WSZ-0610) to 18.0 $\mathrm{hm}^{2}$ (3WQF120-12), which was not a satisfactory result. Obviously, it is necessary to further strengthen the efficiency of UAVs.

Table 5 Percentages of operation items and daily operated

\begin{tabular}{ccccccc}
\multicolumn{7}{c}{ area } \\
\hline UAV & $\begin{array}{c}\text { Preparation } \\
/ \%\end{array}$ & $\begin{array}{c}\text { Failure } \\
\text { rates } \\
/ \%\end{array}$ & $\begin{array}{c}\text { Rout } \\
\text { planning } \\
/ \%\end{array}$ & $\begin{array}{c}\text { Ground } \\
\text { service } \\
/ \%\end{array}$ & $\begin{array}{c}\text { Net } \\
\text { operation } \\
/ \%\end{array}$ & $\begin{array}{c}\text { Area } \\
/ \mathrm{hm}^{2} \cdot \mathrm{d}^{-1}\end{array}$ \\
\hline 3WQF120-12 & 9.56 & 3.73 & 9.75 & 47.03 & 29.93 & 18.0 \\
3CD-15 & 7.60 & 3.42 & 9.84 & 52.14 & 27.00 & 16.7 \\
WSZ-0610 & 8.37 & 4.36 & 10.37 & 48.96 & 27.94 & 13.4 \\
HY-B-15L & 8.81 & 4.17 & 10.26 & -- & -- & -- \\
\hline
\end{tabular}

\section{Conclusions}

In this study, four typical UAVs were used for pesticide application in wheat field. The uniformity of deposition, spraying coverage, droplets density, penetrability and work efficiency of UAVs were tested in this research. The results are showed as follows:

1) Pesticide application of UAVs is on a low precision level, and the spraying liquid distribution was un-uniformity; the deposition performances need to be further improved in China. With the same spray volume and similar operating parameters, the VMD and droplet density showed a negative correlation, while there was no such correlation between the percentages of coverage and VMD.

2) The downward air flow produced by rotors of UAV can promote the spraying liquid deposit into bottom of canopy and increase penetrability.

3) During the whole working time, failure rate was lower than 5\%. The time of preparation, rout planning, ground service took up the most of the whole working time. The rate of net operation was lower than $30 \%$.
4) On the whole, the advantage of high efficiency of UAVs is not fully achieved, and the daily operated area of UAVs is not in a satisfactory level and should be further strengthened.

\section{Acknowledgements}

This work was financially supported by the Special Fund for Agro-scientific Research in the Public Interest (201503130), the National Key Research and Development Plan (Grant No. 2016YFD0200700), National Science Fund Projects (31470099), and Beijing Science and Technology Plan Projects (No. D171100002317003). The authors would like to give special thanks to Anyang Quanfeng Aviation Plant Protection Technology Co., Ltd., Wuxi Hanhe Aviation Technology Co., Ltd., Shandong Wish Plant Protection Machinery Co., Ltd., Shenzhen high-tech New Agricultural Technology Co., Ltd. for their work and cooperation.

\section{[References]}

[1] Liu J. The study about appliance and technology of Cannon rotary-atomizing long-shot air-assisted spraying. MS dissertation. Yangling: Northwest Sci-Tech University of Agriculture and Forestry, 2004. (in Chinese)

[2] Fan Q N. The research on the pesticide spray system using for the mini unmanned helicopter. MS dissertation. Nanjing: Nanjing Forestry University, 2011. (in Chinese)

[3] He X K. Improving severe draggling actuality of plant protection machinery and its application techniques. Transactions of the CSAE, 2004; 20(1): 13-15. (in Chinese)

[4] Qin W C, Qiu B J, Xue X Y, Chen C, Xu Z F, Zhou Q Q. Droplet deposition and control effect of insecticides sprayed with an unmanned aerial vehicle against plant hoppers. Crop Protection, 2016; 85: 79-88.

[5] Faiçal B S, Costa F G, Pessin G, Ueyama J, Freitas H, Colombo A, et al. The use of unmanned aerial vehicles and wireless sensor networks for spraying pesticides. Journal of Systems Architecture, 2014; 60(4): 393-404.

[6] Huang Y B, Hoffmann W C, Lan Y B, Wu W, Fritz B K. Development of a spray system for an unmanned aerial vehicle platform. Applied Engineering in Agriculture, 2008; 25(6): 803-809.

[7] Ru Y, Jin L, Jia Z C, Bao Y, Qian X D. Design and experiment on electrostatic spraying system for unmanned aerial vehicle. Transactions of the CSAE, 2013; 29(24): 1-10. (in Chinese) 
[8] Zhang D Y, Lan Y B, Chen L P, Wang X, Liang D. Current status and future trends of agricultural aerial spraying technology in China. Transactions of the CSAM, 2014; 45(10): 53-59. (in Chinese)

[9] Xue X Y, Tu K, Qin W C, Lan Y B, Zhang H H. Drift and deposition of ultra-low altitude and low volume application in paddy field. Int J Agric \& Biol Eng, 2014; 7(4): 23-28.

[10] Zhang J, He X K, Song J L, Zeng A, Zeng A, Liu Y, et al. Influence of spraying parameters of unmanned aircraft on droplets deposition. Transactions of the CSAM, 2012; 43(12): 94-96. (in Chinese)

[11] Ru Y, Zhou H P, Jia Z C, Wu X W, Fan Q N. Design and application of electrostatic spraying system. Journal of Nanjing Forestry University: Natural Science Edition, 2011; 35(1): 91-94. (in Chinese)

[12] Qiu B J, Wang L W, Cai D L, Wu J H, Ding G R, Guan X P. Effect of flight altitude and speed of unmanned helicopter on spray deposition uniform. Transactions of the CSAE, 2013; 29(24): 25-32. (in Chinese)

[13] Gao Y Y, Zhang Y T, Zhao Y C, Li X H, Yang D B, Yuan H Z. Primary studies on spray droplet distribution and control effects of aerial spraying using unmanned aerial vehicle (UAV) against the corn borer. Plant Protection, 2013; 39(2): 139-142.

[14] Qin W C, Xue X Y, Zhou L X, Zhang S C, Sun Z, Kong W, et al. Effects of spraying parameters of unmanned aerial vehicle on droplets deposition distribution of maize canopies. Transactions of the CSAE, 2014; 30(5): 50-56. (in Chinese)

[15] Wen S, Lan Y B, Zhang J T, Li S H, Zhang H Y, Xing H. Analysis and experiment on atomization characteristics of ultra-low-volume swirl nozzle for agricultural unmanned aviation vehicle. Transactions of the CSAE, 2016; 32(20): 85-93. (in Chinese)

[16] Ru Y, Zhu C Y, Bao R, Li Z F, Ding T. Droplet size distribution of aerial nozzle for plant protection in wind tunnel and flight conditions. Transactions of the CSAE, 2016; 32(20): 94-98. (in Chinese)

[17] Wang C L, He X K, Liu Y J. The small single- and multi-rotor unmanned aircraft vehicles chemical application techniques and control for rice fields in China. Aspects of Applied Biology, 2016; 132: 73-81.

[18] Zhu H, Salyani M, Fox R D. A portable scanning system for evaluation of spray deposit distribution. Computers \& Electronics in Agriculture, 2011; 76(1): 38-43.
[19] Zhang D Y, Chen L P, Zhang R R, Xu G, Lan Y B. Evaluating effective swath width and droplet distribution of aerial spraying systems on M-18B and Thrush 510 G airplanes. Int J Agric \& Biol Eng, 2015; 8(2): 21-30.

[20] Smith D B. Uniformity and recovery of broadcast sprays using fan nozzles. Transactions of the ASAE, 1992; 35(1): 39-44.

[21] GB/T 24677-1-2009. Boom sprayer-Technical requirements.

[22] Zhang P, Deng L, Lyu Q, He S L, Yi S L, Liu Y D, et al. Effects of citrus tree-shape and spraying height of small unmanned aerial vehicle on droplet distribution. Int J Agric \& Biol Eng, 2016; 9(4): 45-52.

[23] Bird S L, Esterly D M, Perry S G. Off-target deposition of pesticides from agricultural aerial spray applications. Journal of Environmental Quality, 1996; 25(5): 1095-1104.

[24] Lan Y, Hoffmann W C, Fritz B K, Martin D E, Lopez J D. Spray drift mitigation with spray mix adjuvants. Applied Engineering in Agriculture, 2008; 24(1): 5-10.

[25] Jaeken P, de Maeyer L, Broers N, Creemers P. Nozzle choice and its effect on spray deposit \& distribution, uptake, drift and biological efficacy in standard apple orchards (Malus sylvestris, $\quad \mathrm{cv} \quad$ Jonagold). Pflanzenschutz Nachrichten Bayer: English Edition, 2003; 56: 326-353.

[26] Wenneker M, Heijne B, van de Zande J C. Effect of air induction nozzle (coarse droplet), air assistance and one-sided spraying of the outer tree row on spray drift in orchard spraying. Annual Review of Agricultural Engineering, 2005; 4(1): 116-128.

[27] Xu X M, Wu P H, Thorbek P, Hyder K. Variability in initial spray deposit in apple trees in space and time. Pest Management Science, 2006; 62(10): 947-956.

[28] Rawn D F K, Quade S C, Shields J B, Conca G, Sun W F, Lacroix G M A, et al. Variability in captan residues in apples from a Canadian orchard. Food Additives \& Contaminants, 2007; 24(2): 149-55.

[29] Ferguson J C, O’Donnell C C, Chauhan B S, Adkins S W, Kruger G R, Wang R B, et al. Determining the uniformity and consistency of droplet size across spray drift reducing nozzles in a wind tunnel. Crop Protection, 2015; 76: 1-6.

[30] Xu B, Chen L P, Tan Y, Xu M. Path planning based on minimum energy consumption for plant protection UAVs in sorties. Transactions of the CSAM, 2015; 46(11): 36-42. (in Chinese) 